\title{
An Application of Distributed Simulation for Hybrid Modeling of Offshore Wind Farms
}

\author{
Navonil Mustafee \\ University of Exeter \\ CISR, Business School \\ +44 (0) 1392725661 \\ n.mustafee@exeter.ac.uk
}

\author{
M'Hammed Sahnoun \\ IRISE Laboratory - CESI \\ Rouen, France \\ +33 (0) 673642966 \\ msahnoun@cesi.fr
}

\author{
Andi Smart, Phil Godsiff \\ University of Exeter \\ CISR, Business School \\ p.a.smart@exeter.ac.uk \\ phil.godsiff@exeter.ac.uk
}

\begin{abstract}
The work in progress paper presents a case study on $M \& S$ of offshore wind farms. Two simulation models have been developed, an agent-based model and a discrete-event model, with the former modeling turbine failures using a degradation function and the latter modeling the Maintenance, Repair and Operations (MRO) strategies. The models have been implemented in NetLogo and Simul8 respectively. In this paper we present ongoing work in hybrid simulation which uses the IEEE 1516 HLA standard for distributed simulation for synchronized model execution and dynamic information exchange between the agent-based and the discrete-event models.
\end{abstract}

\section{Categories and Subject Descriptors}

I.6.3 [Simulation and Modeling]: Applications; I.6.5 [Simulation and Modeling]: Model Development modeling methodologies; I.6.8 [Simulation and Modeling]: Types of Simulation - discrete event, distributed.

\section{General Terms}

Management, Design, Experimentation

\section{Keywords}

Hybrid Simulation; Distributed Simulation; Maintenance, Repair and Operations; Offshore Wind Farm.

\section{INTRODUCTION}

Modelling \& Simulation (M\&S) techniques have their underlying theoretical and methodological foundations which make them relevant for application in specific problem context. However, as the system being modelled increases in complexity it is arguable that researchers need to explore opportunities for the combined application of simulation techniques, or hybrid simulation, to potentially enable synergies across techniques and provide greater insights to problem solving. A profiling study consisting of 525 papers published in the journal Simulation: Transactions of the SCS identified only thirteen studies that have applied

Permission to make digital or hard copies of part or all of this work for personal or classroom use is granted without fee provided that copies are not made or distributed for profit or commercial advantage and that copies bear this notice and the full citation on the first page. Copyrights for third-party components of this work must be honored. For all other uses, contact the Owner/Author.

Copyright is held by the owner/author(s).

SIGSIM-PADS '15, June 10-12, 2015, London, United Kingdom

ACM 978-1-4503-3583-6/15/06.

http://dx.doi.org/10.1145/2769458.2769492 multiple techniques to the problem context; the number of M\&S studies in which the techniques were applied symbiotically were only eight [2]. Our work in progress (WIP) is on the application of hybrid $M \& S$ in the domain of Maintenance, Repair and Operations (MRO) in offshore wind farms. The remainder of our WIP paper is structured as follows. In the next section we provide a definition of hybrid simulation looking at it from both discrete and continuous paradigms. We provide a short description of our application area and the Agent-based Simulation (ABS) and the Discrete-Event Simulation (DES) models that have been developed; we outline the inter-model interaction that will be required to enable hybrid simulation. The following section present a short review on the approaches considered for enabling such simulation using models developed in commercial software packages, and argues for the use of the IEEE 1516 HLA standard for distributed simulation. The final section of the paper describes future work.

\section{HYBRID SIMULATION}

How do we define hybrid simulation? Indeed, the combination of continuous and discrete models has been referred to as hybrid in several studies. We take a wider view of hybrid simulation to also include the methodological aspects of modeling techniques and the advantages gained through the symbiosis of such techniques. For example, ABS and DES are discrete-event but they are also an example of hybrid when they are applied together for the purposes of systems' enquiry. DES relies on the concept of queues and servers, ABS relies on the interaction between individual agents and patterns and behaviors emerge which were not explicitly programmed. Each methodology has its strengths and our definition of hybrid simulation includes investigation of systems which have used multiple simulation techniques, and is thus wider than the notion of only discrete and continuous time. Similarly, the use of System Dynamics (SD; continuous time) with ABS or DES is also an example of hybrid simulation since the application of SD-DES, SD-ABS or indeed SD-DES-ABS will combine the advantages associated with both continuous and discrete models.

\section{M\&S OF OFFSHORE WIND FARMS}

An offshore wind farm is defined as a collection of wind turbines and associated equipment to generate electricity from wind power. In terms of installed capacity, in 2011 Europe had more than $90 \%$ of the world's installed capacity in offshore wind energy (European Wind Energy Association; http://www.ewea.org/). A major obstacle to the ongoing development of this source of energy is the high cost of installation, operation and maintenance compared with other sources of energy [4]. As a result, it is estimated that the cost of maintaining offshore wind turbines makes up between $25 \%-40 \%$ of the total $\mathrm{kWh}$ cost of electricity, compared with $10 \%-15 \%$ of onshore terrestrial sites. Reducing 
maintenance costs through intelligent MRO strategies is a key step in establishing the future of offshore wind farms. Further, modeling of turbine degradation is equally important as factors such as wind speed, events like lightning strike on a turbine, etc. determine the health of the assets and consequently the frequency of maintenance.

\subsection{Modeling Turbine Degradation using ABS}

Through the use of ABS the modeler can model each agent in the system independently, and subsequently add the interactions and relationships between the different parts of the system [1]. The purpose of our ABS model is to consider each wind turbine as an independent agent that is able to interact with its environment, which is composed of the other agents (turbines) and the maintenance and production facilities. Each agent is characterized by variables which represent the state of the turbine it models, its Equipment Health Factor (EHF) and the energy that it produces. Each agent follows two rules - turbine degradation and turbine production - which are influenced by several internal variables (e.g., regular maintenance performed on a turbine will lower turbine degradation, random mechanical outages will not only degrade the performance but also affect energy production) and external variables (e.g., wind speed will determine the energy that is being produced; weather conditions will have a direct bearing to whether maintenance operations can be performed, which in turn is related to turbine degradation). Our ABS model is implemented in NetLogo.

\subsection{Modeling MRO strategies using DES}

Maximization of turbine capability time through intelligent MRO strategies is a crucial operations management activity for both operators of the wind farms (to maximize return on investment) and the turbine manufacturers (to understand turbine performance under actual conditions). The MRO strategies should take into consideration the fact that maintenance activities are dependent on weather windows being available, for example, permissible wave height, wind speed, visibility are all important factors since that determine whether offshore maintenance can take place. The purpose of our DES model is to experiment with various MRO strategies which increase up-time of the wind turbines, while also trying to reduce costs associated with such operations. Our model has been developed using Simul8. The model simulates the operational processes starting from specific faults being reported by individual turbines (includes preventive maintenance) and through until the faults have been rectified and the resources are released for subsequent maintenance operations. The resources in question are the parts for maintenance, the availability of technicians/engineers who would carry out the MRO activities, the availability of the right size of boat (for example, a large boat is required if the maintenance requires the transportation of large spare parts) and the correct mix of these resources for specific MRO tasks.

\subsection{ABS-DES Model Interaction}

Our models have been developed such that the ABS model feeds information on turbine $E H F$ (refer to section 3.1) through simulating a degradation model of individual turbines to the DES model. The DES will then use this information to prioritize maintenance operations in the wind farm; it will simulate several MRO strategies and feed data back to the ABS model to inform that maintenance operations have taken place on specific turbines. The ABS model will then receive this information and will change the EHF for the turbines in question to the maximum permissible value (as good as new). This process repeats itself through the entire simulation timeframe.

\section{EXISITNG APPROACHES}

In a previous study [3] the authors identified three predominant approaches for enabling interaction between models developed using different $M \& S$ methodologies. The first strategy is the manual execution of the models, with the modeler being responsible for transferring variables between them. The second strategy is similar to the previous in respect of the models running independent of each other and demonstrating a similar throttled execution behavior (non-synchronized). However, it is different compared to the first case because this process is now automated. The third strategy of hybrid model execution involves the use of a simulation package like AnyLogic which has built-in support for multi-paradigm M\&S. We have also reviewed wider literature on modelling methodology and have identified the field of distributed simulation to hold promise for our hybrid model execution [3]. Distributed simulation implements well-known time management algorithms to achieve synchronization between individual running simulations. Like our hybrid model, in a distributed simulation the system being modelled may be composed of a number of sub-models, each of which may be mapped to a specific simulation package (in our case this is NetLogo and Simul8). This has been our preferred option.

\section{FUTURE WORK}

The execution strategy for our hybrid M\&S study requires the application of the IEEE1516 HLA standard and the RTI software that implements this standard. The authors are presently implementing a distributed simulation federation which comprises of the ABS and the DES federate, thus also realizing hybrid simulation. The use of the RTI middleware, together with integration software being coded by the authors, will enable our two federates to execute concurrently and without causality and serve as a platform for data exchange.

\section{ACKNOWLEDGEMENTS}

Acknowledgement is made to European Union for the support of this research through the European Program INTERREG IVA France-Channel-UK by funding project entitled MER Innovate.

\section{REFERENCES}

[1] Dimeas, A.L. and Hatziargyriou, N.D. 2005. Operation of a multiagent system for microgrid control. IEEE T. Power Syst. 20, 3, 1447-1455.

[2] Mustafee, N., Katsaliaki, K., Fishwick, P., and Williams, M.D. 2012. SCS - 60 years and counting! A time to reflect on the society's scholarly contribution to M\&S from the turn of the millennium. Simul-T. Soc. Mod. Sim. 88, 9, 10471071 .

[3] Mustafee, N., Sahnoun, M., Smart, A., et al. 2015. Investigating Execution Strategies for Hybrid Models developed using Multiple M\&S Methodologies. In Proceedings of the 2015 Spring Simulation MultiConference (Alexandria, VA, April 12-15, 2015). ACM.

[4] Pineda, I., Azau, S., Moccia, J., and Wilkes, J. 2014. Wind in power - 2013 European statistics. The European Wind Energy Association, 1-12. 